Www.jmscr.igmpublication.org

Impact Factor (SJIF): 6.379

Index Copernicus Value: 79.54

ISSN (e)-2347-176x ISSN (p) 2455-0450

crossrefDOI: https://dx.doi.org/10.18535/jmscr/v6i8.103

Journal Of Medical Science And Clinical Research

\title{
Rectal Versus Intramuscular Diclofenac Sodium for Pain Relief Following Caesarean Section: A Randomised Controlled Trial
}

Authors

\author{
Otutoaja Uzoma ${ }^{1}$, Adeniyi Augustine Adebayo ${ }^{1}$, Bakare Adewumi ${ }^{1}$, \\ Orewole Olayinka Tesleem², Adewara Emmanuel Olumide ${ }^{1}$,
} Okere Raymond Akujuobi ${ }^{1}$, Awoyinka Babatunde ${ }^{1}$, Adebara Idowu Oluseyi ${ }^{1}$, Adeyemo Timothy Olabisi ${ }^{1}$, Ayankunle Mojisola $\mathrm{O}^{1}$, Busari Olusegun ${ }^{3}$

${ }^{1}$ Department of Obstetrics and Gynaecology, Afe Babalola University, Ado Ekiti/Federal Teaching Hospital, Ido- Ekiti, Nigeria

${ }^{2}$ Department of Anaesthesia, Afe Babalola University, Ado Ekiti/ Federal Teaching Hospital, Ido- Ekiti, Nigeria

${ }^{3}$ Department of Medicine, Afe Babalola University, Ado Ekiti/Federal Teaching Hospital, Ido- Ekiti, Nigeria

Corresponding Author

Adeniyi Augustine Adebayo

Department of Obstetrics and Gynaecology, Afe Babalola University, Ado Ekiti/Federal Teaching Hospital, Ido- Ekiti, Nigeria

Phone number: +234 8037141492, Email: adeniyiaa@abuad.edu.ng; bayoadeniyis@gmail.com

\begin{abstract}
Introduction: Patients who undergo caesarean delivery should have adequate postoperative pain relief so as to ensure maternal and neonatal wellbeing. Management of postoperative pain after caesarean section requires a balance between pain relief and undesirable side effects of drugs and route of administration. This study was designed to compared the analgesic efficacy of rectal diclofenac and intramuscular diclofenac for pain relief following caesarean section.

Method: A total number of sixty six (66) patients undergoing elective lower segment caesarean delivery under spinal anaesthesia were randomized into two groups. Thirty-three (33) patients in each group received either $100 \mathrm{mg}$ of rectal diclofenac sodium or $75 \mathrm{mg}$ of intramuscular diclofenac sodium for duration of 24 hours, following caesarean section.

Results: Fewer patients in the rectal arm had rescue analgesia,12(42.86\%) vs $16(57.14 \%), P=0.21$. The number of rescue analgesia was also lower in the rectal group, 16(41.03\%) vs 23(58.97\%), $P=0.027$. The time interval between commencement of post-operative analgesia and demand for rescue analgesic agent was similar in both group $4.28 \pm 2.78$ hours and $3.98 \pm 2.52$ hours respectively, $P=0.77$. The pain scores were higher in the intramuscular diclofenac group at 6, 12, 18 and 24 hours post operation. Side effects occur more frequently in the intramuscular group than in the rectal group but patients' satisfaction was similar in both groups, rectal (84.8\%) and intramuscular (78.8\%), $P=0.612$.

Conclusion: Both routes of administration demonstrate good pain control, however rectal route appears more tolerable among the patients.
\end{abstract}

Keywords: Post Caesarean pain, Diclofenac, administration. 


\section{Introduction}

Caesarean section commonly induces moderate to severe pain for about 24 to 48 hours $^{1}$. It requires optimal perioperative pain management; which not only allows the mother to ambulate early but also plays a key role in the postoperative rehabilitation, relieves suffering, leads to shortened hospital stay, reduced hospital costs, and increased patient satisfaction ${ }^{2}$. Though different approaches have been introduced for proper pain relief, but these multimodal approaches are still inadequate and unsatisfactory in many patients ${ }^{3,4,5}$.

Several studies have investigated different protocols of post-partum pain management in women undergoing caesarean section and some new technologies of postoperative pain treatment have also been reported ${ }^{6}$. The Royal College of Anaesthetists proposed standards for post caesarean section pain relief, which includes that $>90 \%$ of women should have a worst pain score of $<3$ on a visual analogue scale of $0-10^{7}$, Nonsteroidal anti-inflammatory drugs should be prescribed to $100 \%$ of women and $>90 \%$ of women should be satisfied with pain management ${ }^{8}$.

To reduce postoperative pain, different drugs including opioids and non-steroidal antiinflammatory drugs (NSAIDs) have been used. In our institution, the standard protocol for analgesia following caesarean section involves the use of diclofenac and pentazocine, both given intramuscularly. Maternal satisfaction from this protocol and the use of rectal analgesia has not been previously studied. Also, the use of opioids is associated with side effects such as nausea, vomiting, confusion, constipation, tolerance and physical dependency ${ }^{9,10,11}$.

For this reason, NSAIDs, especially in the form of suppository, have been taken into consideration with pentazocine (opioid) used only as a rescue analgesic agent. Though the mechanism of action of the two analgesic agents: rectal diclofenac sodium and intramuscular diclofenac sodium are similar, the advantages of using suppository include their ease of administration, painless application and there is no need for injection devices. ${ }^{12,13,14}$. This study was designed to compare the relief of pain following caesarean section using similar analgesic agents, but different routes of administration.

\section{Materials and Methods}

This was a randomized controlled study comparing the effectiveness of rectal diclofenac sodium and intramuscular diclofenac sodium for pain control following caesarean section among pregnant women who were scheduled for elective caesarean section under spinal anaesthesia. Sixtysix (66) women who satisfied the inclusion criterion were recruited. Exclusion criteria included but limited to, morbid obesity, coagulopathy, pre-eclampsia and conditions exacerbated by NSAIDs such as peptic ulceration. The 66 women were recruited based on the minimum calculated sample size of 25 patients on each arm of the study and deliberate over sampling of $25 \%$. This gave the study a statistical power of $80 \%$ and level of significance of 0.05 at 95\% confidence level. The patients were randomized with the aid of computer generated random numbers into two groups. Thirty -three (33) women on each arm of the study received either $100 \mathrm{mg}$ rectal diclofenac every 12 hours or $75 \mathrm{mg}$ intramuscular diclofenac every 12 hours for 24 hour on both arms of the study.

On admission of patients for elective caesarean delivery, those that met the inclusion criteria were recruited into the study. Consent for study participation was taken and the respondent assigned a sequential study number. The most senior doctor or nursing staff on duty, responsible for maintaining the envelopes was then contacted and the corresponding numbered envelope was opened for the purpose of allocation into the rectal or intramuscular diclofenac arms of the study. All patients recruited for the study had caesarean section under spinal anaesthesia using the same local anaesthetic agent, 3mls of $0.5 \%$ (15mg) Bupivacaine hydrochloride. 
The first dose of diclofenac was administered immediately after vulvo-vaginal toileting while patient was still on the operating table and then 12hourly for 24 hours in the postnatal ward, either rectally by gently parting the buttocks or through intramuscular route into the upper outer quadrant of the buttocks deep into the gluteus muscle. The degree of response to post operative pain was monitored and assessed by visual analogue scale, rated zero (0) to ten (10) on a chart, with 0 representing no pain and 10 representing unbearable pain. The post-operative pain was then scored as mild when the patient marked any point on the chart from 0-3, moderate when she indicated any point from 4-6 and severe on indication of any point from 7-10.

Intramuscular pentazocine was used as rescue analgesia at a dose of $30 \mathrm{mg}$; this was given when the pain score on the visual analogue scale was 7 or above. The time of administration of rescue analgesia was noted as well as the doses of rescue analgesia administered to the subjects in the different study arms within the period of 24 hours. The women were also asked to express their overall satisfaction with the analgesic method during the post-operative period of 24 hours. Postoperative pain score was assessed by one of the members of the research team who was not aware of the route of drug administration a patient was randomized to receive.

\section{Data Analysis}

The data generated were analyzed using the Statistical Package for Social Science (SPSS) for Windows software version 17. Frequency tables were made and the results tested for statistical significance. Results were presented as mean \pm standard deviation. Student's $t$-test was used to determine the differences between the means of the two groups. For comparing categorical data, Chi square test and Fisher's exact test were used when appropriate. The level of statistical significance was set at $p$-value $<0.05$.

\section{Results}

The important demographic characteristics were similar in both groups, the clinical and the obstetric parameters were also similar (Table 1 and 2). The number of patients who had rescue analgesia were lower in the rectal arm compared to the intramuscular arm, $12(42.86 \%)$ vs 16 $(57.14 \%)$, this was however not statistically significant $(\mathrm{P}=0.21)$. The total number of doses of rescue analgesic agent administered was also lower in the rectal group when compared to the intramuscular group, 16(41.03\%) vs 23(58.97\%), this was also not statistically significant $(\mathrm{P}=0.027)$. The time interval between commencement of post-operative analgesia and demand for rescue analgesic agent as well as maternal expression of satisfaction following use of either route of analgesia was found not to be statistically significant (Table 3).

Although most respondents recorded a score within the range of mild to moderate pain at different stages of the study, Overall, the mean pain scores were higher in the intramuscular diclofenac group at $6,12,18$ and 24 hours post operation, however these did not assume statistical significance $(\mathrm{p}>0.05)$ (Table 4)

All the five reported adverse effects (nausea, vomiting, dizziness and headaches) were in the intramuscular route arm of the study (Fig. 1) 
Table 1: Socio-demographic characteristics of respondents

\begin{tabular}{|c|c|c|c|c|}
\hline & Rectal & Intramuscular & Statistical test & P value \\
\hline Maternal Age (Years) & $\mathrm{n}(\%)$ & $\mathrm{n}(\%)$ & \multirow[b]{7}{*}{$0.780 *$} & \multirow[b]{7}{*}{0.12} \\
\hline$<20$ & $0(0.0)$ & $2(6.1)$ & & \\
\hline $21-25$ & $1(3.0)$ & $5(15.1)$ & & \\
\hline $26-30$ & $12(36.4)$ & $10(30.3)$ & & \\
\hline Above 30 & $20(60.6)$ & $16(48.5)$ & & \\
\hline Total & 33(100.0) & 33(100.0) & & \\
\hline Mean \pm SD & $32.39 \pm 4.64$ & $38.48 \pm 4.60$ & & \\
\hline Educational Level & $\mathbf{n}(\%)$ & n(\%) & \multirow{6}{*}{$1.010 * *$} & \multirow{6}{*}{0.79} \\
\hline None & $2(6.1)$ & $1(3.0)$ & & \\
\hline Primary & $1(3.0)$ & $2(6.1)$ & & \\
\hline Secondary & $9(27.3)$ & $10(30.3)$ & & \\
\hline Tertiary & $21(63.6)$ & $20(60.6)$ & & \\
\hline Total & 33(100.0) & 33(100.0) & & \\
\hline Marital status & $\mathrm{n}(\%)$ & $\mathrm{n}(\%)$ & \multirow{4}{*}{$2.276 * *$} & \multirow{4}{*}{0.98} \\
\hline Married & $32(97.0)$ & $31(93.9)$ & & \\
\hline Single & $1(3.0)$ & $2(6.1)$ & & \\
\hline Total & 33(100.0) & 33(100.0) & & \\
\hline Religion & $\mathrm{n}(\%)$ & $\mathrm{n}(\%)$ & \multirow{4}{*}{$1.025^{* *}$} & \multirow{4}{*}{0.76} \\
\hline Islam & $5(15.2)$ & $4(12.1)$ & & \\
\hline Christianity & $28(84.8)$ & $29(87.9)$ & & \\
\hline Total & 33(100.0) & 33(100.0) & & \\
\hline Tribe & $\mathrm{n}(\%)$ & $\mathrm{n}(\%)$ & \multirow{6}{*}{$2.820 * *$} & \multirow{6}{*}{0.80} \\
\hline Igala & $1(3.0)$ & $0(0.0)$ & & \\
\hline Yoruba & $30(91.0)$ & $29(87.9)$ & & \\
\hline Igbo & $0(0.0)$ & $4(12.1)$ & & \\
\hline Bini & $2(6.0)$ & $0(0.0)$ & & \\
\hline Total & 33(100.0) & 33(100.0) & & \\
\hline Occupation & $\mathrm{n}(\%)$ & $\mathrm{n}(\%)$ & \multirow{7}{*}{$3.423^{* *}$} & \multirow{7}{*}{0.43} \\
\hline Trading & $12((36.4)$ & $7(21.2)$ & & \\
\hline Teaching & $14(42.4)$ & $17(51.6)$ & & \\
\hline Artisans & $5(15.2)$ & $2(6.0)$ & & \\
\hline Nursing & $1(3.0)$ & $3(9.1)$ & & \\
\hline Unemployed & $1(3.0)$ & $4(12.1)$ & & \\
\hline Total & $33(100.0)$ & 33(100.0 & & \\
\hline
\end{tabular}

Table 2 Clinical and Obstetric characteristics of respondents

\begin{tabular}{|c|c|c|c|c|}
\hline \multirow{2}{*}{$\begin{array}{ll}\text { Clinical and } \\
\text { characteristics }\end{array}$} & Rectal & Intramuscular & Statistical test & \multirow{2}{*}{$P$ value } \\
\hline & Mean \pm SD & Mean \pm SD & $\mathrm{T}$ test & \\
\hline Maternal weight $(\mathrm{Kg})$ & $74.63 \pm 9.07$ & $76.76 \pm 8.12$ & -1.232 & 0.221 \\
\hline \multirow[t]{2}{*}{ Gestational age at delivery (wks) } & $38.42 \pm 1.34$ & $37.90 \pm 1.50$ & -1.826 & 0.071 \\
\hline & $\mathrm{n}(\%)$ & $\mathrm{n}(\%)$ & Fisher's exact test & \\
\hline Booked & $32(97.0)$ & $31(93.9)$ & \multirow{2}{*}{0.219} & \multirow{2}{*}{0.640} \\
\hline Unbooked & $1(3.0)$ & $2(6.1)$ & & \\
\hline Primigravidae & $1(3.0)$ & $4(12.1)$ & \multirow{2}{*}{0.271} & \multirow{2}{*}{0.603} \\
\hline Multigravidae & $32(97.0)$ & $29(87.9)$ & & \\
\hline
\end{tabular}


Table 3 Rescue analgesia administration

\begin{tabular}{|l|c|c|c|c|}
\hline & $\begin{array}{c}\text { Rectal } \\
\mathbf{n}(\%)\end{array}$ & $\begin{array}{c}\text { Intramuscular } \\
\mathbf{n}(\%)\end{array}$ & $\begin{array}{c}\text { Statistical } \\
\text { test }\end{array}$ & $\begin{array}{c}\text { P } \\
\text { value }\end{array}$ \\
\hline No of patients who had rescue analgesia & $12(42.86 \%)$ & $16(57.14 \%)$ & $\mathrm{X}^{2}=1.57$ & 0.21 \\
\hline $\begin{array}{l}\text { Total number of doses of rescue analgesic } \\
\text { agent administered }\end{array}$ & $16(41.03 \%)$ & $23(58.97 \%)$ & & \\
\hline Mean \pm SD & $1.46 \pm 0.52$ & $1.44 \pm 0.63$ & $\mathrm{t}=2.35$ & 0.027 \\
\hline Median & 1 & 1 & & \\
\hline Range & 1 & 2 & & \\
\hline $\begin{array}{l}\text { Time interval between commencement of } \\
\text { post-operative analgesia and demand for } \\
\text { rescue analgesia agent(hrs) }\end{array}$ & & & & \\
\hline Mean \pm SD & $4.28 \pm 2.78$ & $3.98 \pm 2.52$ & $\mathrm{t}=-0.29$ & 0.77 \\
\hline Median & 4.0 & 3.5 & & \\
\hline Range & 8.67 & 9.25 & & \\
\hline Maternal expression of satisfaction & & & & \\
\hline Yes & $28(84.8)$ & $26(78.8)$ & $\mathrm{X}^{2}=0.257$ & 0.612 \\
\hline No & $5(15.2)$ & $7(21.2)$ & & \\
\hline
\end{tabular}

$t$ - independent $t$ test, $X^{2}$ - Chi-square test

Table 4 Comparison of pain scores at 6, 12, 18, 24-hour post-caesarean section

\begin{tabular}{|l|c|c|c|c|}
\hline & Rectal & Intramuscular & T-test & P value \\
\hline 6 hours post operation & & & & \\
\hline Mean \pm SD & $4.52 \pm 1.46$ & $4.58 \pm 1.82$ & 0.15 & 0.88 \\
\hline Median & 5 & 4 & & \\
\hline Range & 7 & 9 & & \\
\hline 12 hours post operation & & & & 0.28 \\
\hline Mean \pm SD & $4.12 \pm 1.56$ & $4.52 \pm 1.39$ & 1.08 & \\
\hline Median & 4 & 4 & & \\
\hline Range & 7 & 8 & & 0.12 \\
\hline 18 hours post operation & & & & \\
\hline Mean \pm SD & $3.24 \pm 1.50$ & $3.79 \pm 1.29$ & 1.58 & \\
\hline Median & 3 & 4 & & \\
\hline Range & 5 & 5 & & \\
\hline 24 hours post operation & & & & \\
\hline Mean \pm SD & $2.88 \pm 1.90$ & $3.76 \pm 1.75$ & 1.95 & \\
\hline Median & 3 & 4 & & \\
\hline Range & 10 & 5 & & \\
\hline
\end{tabular}

\section{Discussion}

This study again confirmed diclofenac as an effective analgesic for post operative pains with less than $50 \%$ of the patients requiring rescue analgesia. The need for rescue analgesia was less in the rectal arm $12(42.86 \%)$ vs $16(57.14 \%)$, $(\mathrm{P}=0.21)$. This was in keeping with similar studies carried out by Olofsson et $\mathrm{al}^{12}$ and Dahl et al ${ }^{85}$ that patients receiving additional analgesia were fewer in the rectal diclofenac group. A total number of 39 doses of rescue analgesia were administered and this was also lower in the rectal group when compared to the intramuscular group, $16(41.03 \%)$ vs $23(58.97 \%)$, and this was statistically significant $(\mathrm{P}=0.027)$. Rashid et $\mathrm{al}^{13}$ had similar finding in which the rectal diclofenac group (study group) had 28 pethidine injections(35\%) and was significantly lower when compared to the 52 pethidine injections $(65 \%)$ in the control group. They concluded that rectal diclofenac reduces opioid requirements with a corresponding reduction in the opioid related side effects. The above finding was also corroborated by Haleh Rahmanpoor et $\mathrm{al}^{77}$ that the rectal diclofenac group didn't need additional analgesia and control of pain in this group was better than the pethidine group. The findings by Cardoso et al in their research was however different. ${ }^{14}$ They found out that the need for rescue analgesia (meperidine) was higher in the rectal diclofenac group as compared to the intramuscular diclofenac group.

The time interval between commencement of post-operative analgesia and demand for rescue analgesic agent was longer in the rectal diclofenac 
arm compared to the intramuscular arm $(4.28 \pm$ $2.78 \mathrm{hrs}$ vs $3.98 \pm 2.52$ ) though not statistically significant $(\mathrm{P}=0.77)$. This was in keeping with the findings by Rashid et al that the addition of a single $100 \mathrm{mg}$ rectal diclofenac after caesarean section increased the mean time to first analgesia by more than 5 hours. ${ }^{13}$ This could possibly be explained by the fact that the rate of absorption of diclofenac from suppositories is slower and has a sustained analgesic effect unlike when administered intramuscularly. ${ }^{78}$

The summary of the pain scores in the first 24 hours post-caesarean section showed that the level of pain perception in both groups were majorly in the mild and moderate range. This confirmed similar studies which revealed that diclofenac is an effective analgesia for the control of postoperative pain. ${ }^{12,77,85,86}$ The progressive reduction of the pain score over the first 24 hours in both groups also confirmed that diclofenac given at repeated doses is effective for post-caesarean section pain control which is usually severe in the first 24 to 48 hours of the puerperium. ${ }^{12,77,85,86}$ This is possibly because the peak plasma concentration of diclofenac is high up to 12 hours post-administration. ${ }^{78}$

However at every 6 hours of assessing the level of pain, using the visual analogue scale, rectal diclofenac produced a lesser pain score compared to intramuscular diclofenac throughout the first 24 hours. This finding though not statistically significant was similar to the study carried out by Rashid et al where they observed that rectal diclofenac is an effective post-operative analgesic as it provided better analgesia at 12, 18 and 24 hours after caesarean section. ${ }^{13}$ Kyomugisha also, after comparing rectal suppository and injectable diclofenac in postoperative pain control following inguinal herniorrhaphy, observed that the postoperative pain intensity scores at 12, 24, 36 and 48 hours were significantly lower in the rectal suppository group. He then concluded that the rectal route of diclofenac as a suppository was more effective than the intramuscular route. ${ }^{63}$
This study reported occurrence of side effects such as nausea, vomiting, headache and dizziness in five of the respondents in the intramuscular diclofenac group and none in the rectal diclofenac group. This was similar to the findings by Rashid et al in their study of the use of rectal diclofenac for post-caesarean analgesia where they recorded no gastrointestinal problems, no bleeding disorder or reports of local irritation from the suppositories. ${ }^{13}$ This was, however, not in keeping with the findings by Surakam et al when they studied the use of iintramuscular diclofenac for analgesia after caesarean delivery. Here, they noticed no side effects of intramuscular diclofenac such as gastrointestinal bleeding, uterine atony or injection site irritation. ${ }^{91}$

Overall, the maternal expression of satisfaction was similar in both study groups, rectal (84.8\%) and intramuscular $(78.8 \%)$. The difference was not statistically significant. This was attributable to the analgesic efficacy of diclofenac as the duration of the therapeutic effect of diclofenac is 3-4 times longer than its half-life in the plasma and release of pain mediating prostaglandins are inhibited and remain so despite the decrease of the drug's plasma concentration. ${ }^{13,85,86}$

In conclusion, the study again reiterate the effectiveness of diclofenac for post caesarean section analgesia, however the rectal route is found to have advantages over the intramuscular route.

\section{Sources of support: Nil}

\section{References}

1. Breivik H, Stubhaug A. Management of acute postoperative pain: still a long way to go! Pain. 2008; 137(2): 233-234.

2. Kuczkowski KM. Postoperative pain control in the parturient: new challenges (and their solutions) J Clin Anesth. 2004; 16(1): 1-3.

3. Pan PH. Post caesarean delivery pain management: multimodal approach. Int $J$ Obstet Anesth. 2006; 15(3): 185-188. 
4. Faboya A, Uncles D. Post Caesarean delivery pain management: multimodal approach. Int J Obstet Anesth. 2007; 16(2): 185-186.

5. Gadsden J, Hart S, Santos AC. Postcaesarean delivery analgesia. Anesth Analg. 2005; 101(5): 62-69.

6. Snell P, Hicks C. An exploratory study in the UK of the effectiveness of three different pain management regimens for post-caesarean section women. Midwifery 2006; 22(3): 249-261.

7. Collins SL, Moore RA, McQuay HJ. The visual analogue pain intensity scale: what is moderate pain in millimeters? Pain 1997; 72: 95-97.

8. McDonnell NJ, Keating ML, Muchatuta NA, Pavy TJ, Paech MJ. Analgesia after caesarean delivery. Anaesth Intensive Care 2009; 37(4): 539-551.

9. Neugebauer E, Wilkinson $\mathrm{R}$, Kehlet $\mathrm{H}$, Schug S. PROSPECT: a practical method for formulating evidence-based expert recommendations for the management of postoperative pain. Surg Endosc. 2007; 21(7): 1047-1053.

10. Rüsch D, Becker K, Eberhart H, "Postoperative Nausea and Vomiting (PONV)—Recommendations for Risk Assessment, Prophylaxis and TherapyResults of an Expert Panel Meeting," Anasthesiologie, Intensivmedizin, Notfallmedizin, Schmerztherapie 2011; 14(5): 158-170.

11. Bonnet M, Mignon A, Ozier $\mathrm{Y}$, "Analgesic Effect and Adverse Effects of Epidural Morphine Compared to Parenteral Opioids after Elective Caesarean Section: A Systemic Review," European Journal of Pain 2010; 14(9): 894-899.

12. Olofsson CI, Legeby MH, Nygards EB, Ostman KM. Diclofenac in the treatment of pain after caesarean delivery: An opioid-saving strategy. Eur. J. Obstet. Gynecol. Reprod. Biol.2000; 88: 143-146.

13. Rashid M, Jaruidi $H$. The use of rectal diclofenac for post-caesarean analgesia. Saudi Med. J. 2000; 21: 145-149.

14. Cardoso M, Carvalho J, Tahamtani S. Intramuscular versus rectal diclofenac associated with low dose spinal morphine for post-caesarean analgesia. Revista Brasileira de Anestesiologia. November 2002; 52(6): 666-672.

15. Dahl V, Hagen IE, Sveen AM, Norseng H, Koss KS, Steen T. High-dose diclofenac for postoperative analgesia after elective caesarean section in regional anaesthesia. Int J Obstet Anesth. 2002;11(2):91-94

16. Haleh R, Seyed N, Fatemeh K. Comparison of Diclofenac with Pethidine on the Pain after Cesarean Section. International Journal of Pharmacology 2007; 3: 201-203.

17. Bertram G, Susan B, Anthony J. Nonsteroidal Anti-inflammatory Drugs. In Basic \& Clinical Pharmacology, 11th Edition. Mc Graw-Hill Companies, 2009.

18. Al-Waili NS1. Efficacy and safety of repeated postoperative administration of intramuscular diclofenac sodium in the treatment of post-cesarean section pain: a double-blind study. Arch Med Res. 2001; 32(2):148-154.

19. Kyomugisha E. Comparison of rectal suppository and injectable diclofenac in postoperative pain control following inguinal herniorrhaphy in Mulago hospital. Makerere University institutional repository 2003; 6: 41-46.

20. Surakarn J, Tannirandorn Y. Intramuscular diclofenac for analgesia after caesarean delivery: a randomized controlled trial. $J$ Med Assoc Thai. 2009; 92(6):733-737. 